Anuario de Psicología

2005, vol. 36, n ${ }^{\circ} 1,37-60$

(C) 2005, Facultat de Psicologia

Universitat de Barcelona

\title{
Análisis de los factores psicológicos moduladores del dolor crónico benigno
}

\author{
Jenny Moix Queraltó \\ Universidad Autónoma de Barcelona
}

Cada día son más abundantes los estudios que muestran que los factores psicológicos juegan un papel clave en el proceso del dolor. Desde el campo del dolor se ha importado el modelo de estrés de Lazarus para el estudio de estos factores psicológicos. Esta teoría sugiere que el estrés que sufre un sujeto ante un evento potencialmente amenazante se debe a cómo lo evalúa y a cómo lo afronta. Traduciendo este modelo a la experiencia del dolor: el estímulo doloroso provocará más o menos discapacidad según cómo sea evaluado y afrontado. En el presente trabajo, realizamos una revisión de los trabajos más actuales en el campo del dolor articulándolos dentro del modelo de Lazarus. En una segunda parte del artículo, describimos las técnicas interdisciplinares que se emplean en el tratamiento del dolor crónico. Por último, animamos a que el modelo de Lazarus se emplee como guía para el diseño de futuras terapias.

Palabras clave: dolor crónico, modelo de Lazarus, evaluación cognitiva, estrategias de afrontamiento, tratamiento interdisciplinar.

A growing body of studies stresses the importance of psychological factors in the pain process. The Lazarus stress model has been transferred to the chronic pain field to study these factors. This model suggests that when there is a potentially stressful event, anxiety levels depend on the cognitive evaluation of pain stimulus and the strategies used to cope with it. Psychological pain factors which have recently appeared in the literature are analysed from the perspective of the Lazarus model. In the second part, interdisciplinary techniques used in the chronic pain field are described. Finally, health workers are encouraged to use the Lazarus model as a guide for the design of future chronic pain therapies.

Key wods: Chronic pain, Lazarus model, cognitive evaluation, coping strategies, interdisciplinary therapy.

\footnotetext{
Agradecimientos: quiero agradecer a Tomás Blasco su atenta revisión del manuscrito.

Correspondencia: Jenny Moix Queraltó. Grupo de Investigación en Estrés y Salud (GIES). Facultad de Psicología. Edificio B. Campus de la UAB. 08193 Bellaterra (Barcelona). Correo electrónico: jenny.moix@uab.es
} 
El dolor crónico es un trastorno que padecen millones de personas en todo el mundo. Concretamente en España, el $23.4 \%$ de la población sufre este trastorno (Català, 2002).

El dolor es sólo uno de los muchos motivos de sufrimiento que viven estas personas dado que el dolor no va solo, sino que trae consigo oscuros sentimientos y emociones. Una dolencia, en principio física, se convierte en psicológica e incluso social dado que el dolor se extiende afectando a las personas más próximas: pareja, familia, amigos...

La repercusión del dolor puede incluso llegar al ámbito económico. En un estudio realizado en España en el que se entrevistaron 2998 personas, se pudo comprobar que el dolor crónico no sólo provoca incapacidad y merma la calidad de vida, sino que ocasiona un importante número de visitas médicas, incapacidad laboral y consumo de medicamentos, consecuencias que tienen una clara incidencia a nivel monetario (Carmona, Ballina, Gabriel y Laffon, 2001).

Como vemos, el dolor parece no tener unos límites claros, así pues para acotar este concepto, el primer paso deberá consistir en definirlo.

\section{¿Cómo podemos definir el dolor?}

La manera como se define un trastorno es determinante, dado que según sea la definición, la problemática se analizará desde una perspectiva u otra, incidiendo, en último término, en la forma en la que se tratará. La definición de dolor ha ido evolucionando a lo largo del tiempo, lo que ha traído consigo un cambio en la forma de estudiarlo y tratarlo.

Las primeras definiciones de dolor que podemos encontrar tienen un carácter marcadamente físico. En ellas se contempla el dolor como una sensación que responde directamente a un daño o agresión tisular. La sensación, en este caso, sería proporcional al daño. Cuanto mayor es la herida, mayor es el dolor. $\mathrm{Si}$ no hay herida, no hay dolor y, por consiguiente, si no hay dolor, no hay herida. gica”.

Actualmente, la definición del dolor es mucho más amplia, más "psicoló-

La Asociación Internacional para el Estudio del Dolor (Cf. González, 1999) lo describe como «Una experiencia sensorial y emocional asociada a una lesión real o potencial». La palabra "emocional" convierte al dolor no sólo en una experiencia fisiológica sino también psicológica, mientras el vocablo "potencial" sugiere que se puede experimentar dolor sin que exista una lesión real. La inclusión de estos dos términos permitiría hablar de dolor en personas en las que no se detecta causa física.

Desafortunadamente, aunque los profesionales que trabajan en dolor suelen "teóricamente" creer en esta visión más amplia -esto es, lo consideran como un fenómeno biopsicosocial-, en realidad, la gran mayoría de ellos utiliza técnicas terapéuticas exclusivamente basadas en principios biomédicos (Thunberg, Carlsson y Hallberg, 2001). De ahí la necesidad no sólo de analizar los factores psicológicos y sociales implicados en dolor, sino sobre todo de proponer técnicas terapéuticas que se deriven de estos análisis. 


\section{¿Cuándo el dolor pasa a considerarse crónico? ¿Qué tipos de dolor crónico existen?}

Un dolor que persista durante más de 3 meses se considera dolor crónico. Dentro del dolor crónico podemos clasificar dos tipos de dolor: maligno y benigno.

El dolor maligno es el que indica que existe un deterioro progresivo en nuestro organismo. El ejemplo más claro sería el dolor oncológico.

El dolor benigno también lo podríamos etiquetar como dolor inútil ya que no "avisa" de ningún deterioro. En muchos casos, se trata de un dolor con causa orgánica que, una vez desaparecida, todavía persiste. Incluidos en esta clase de dolor, podemos encontrar diferentes tipos: cefaleas, fibromialgia, dolor lumbar, neuropatías, etc. Dentro del dolor benigno, en algunos casos, podemos oír el término de dolor psicógeno para referirse a que la causa es enteramente psicológica. El dolor psicógeno es poco frecuente. De hecho, no creemos que la etiqueta de "psicógeno" sea correcta. Al igual que Vallejo y Comeche (1994) consideramos que el calificativo de psicógeno es una cuestión de grado. Esto es, en todo dolor hay un componente psicológico que actúa como modulador. Para nosotros, como veremos a lo largo de este trabajo, lo realmente importante no es la causa inicial del dolor, sino lo que determina la discapacidad provocada por éste y los factores que incidirán en su cronificación. A continuación vamos a ver a qué nos referimos cuando afirmamos que el dolor discapacita; a saber, qué alteraciones psicológicas conlleva.

\section{¿Cuál es el estado psicológico de los pacientes con dolor crónico?}

En muchos casos, el dolor es en algún grado discapacitante y afecta a prácticamente todas las áreas de la vida: laboral, tiempo libre, sexual, sueño... Es usual que las personas se sientan inútiles, su humor se altere y todo ello incida también en los afectos provocando un deterioro en sus relaciones con los demás. No sólo existe una alteración a nivel emocional, sino también a nivel cognitivo (es fácil encontrar deterioros en los procesos atencionales y de memoria).

En el ámbito afectivo, muchos se sienten incomprendidos no sólo por sus allegados, sino también por los profesionales médicos que no saben o no pueden explicarles con claridad su patología. En la mayoría de los casos, se insinúa que su patología es psicológica, lo cual es vivido como si se los tachara de "locos". Con el objetivo de conocer detalladamente qué piensan y sienten los pacientes cuando los profesionales de la medicina dudan respecto a la organicidad de su dolor, May, Rose y Johnstone (2000) entrevistaron a 12 pacientes con dolor lumbar crónico. Los pacientes se resistían a creer que su dolor podía estar influenciado por factores psicológicos y vivían esta insinuación como si se los culpabilizara de su dolor. Estos sujetos trasladaban toda la culpa de su dolor a la incertidumbre que mostraban los profesionales en torno a la etiología y tratamiento de su patología. Según los entrevistados, el escepticismo de los médicos era dañino y descorazonador.

La depresión y la ansiedad son sin duda las dos emociones más usuales entre los pacientes con dolor crónico. En algunos casos, el dolor también puede 
desembocar en agresividad dirigida principalmente contra los profesionales o instituciones médicas (Fishbain, 2000). El dolor no sólo provoca emociones tan negativas como la depresión, la ansiedad o la agresividad, sino que puede incluso producir un trágico final en algunas vidas: el suicidio. Parece que no es el dolor per se el responsable del suicidio, sino que la variable clave que correlaciona en mayor medida con el suicidio es la depresión que experimentan algunos pacientes (Fisher, Haythornthwaite, Heinberg, Clark y Reed, 2002).

El dolor crónico como vemos es algo más, algo muchísimo más complejo que simplemente sentir dolor. En palabras de González (1999):

Imaginen Vds. que pudiésemos, con la fuerza de los pensamientos infantiles, trasformar nuestro bolígrafo en una varita mágica, y del dolor crónico nos fuera posible retirar la parte afectiva, desprender la cuestión beneficio sea buscado o no, descontar las compensaciones sociales y económicas, buscadas o no, ignorar la influencia que ejercen factores externos como calor, frío, cultura, educación, olvidar la innegable influencia del estrés o la depresión, y finalmente pudiésemos apartar otros factores importantes del tipo psiquiátrico, educacional, familiar, etc. ¿Qué nos quedaría? (...) Pues posiblemente sólo una cuestión dolorosa de escasa entidad (González, 1999, 10).

Estas palabras nos muestran innumerables aspectos que son afectados y desencadenados por el dolor. Expresan los sentimientos negativos que provoca. No obstante, ante el sufrimiento del dolor crónico no todas las personas reaccionan igual, ni sienten lo mismo. Mientras algunas caen en una profunda depresión, otras logran adaptar su vida al dolor sin que su estado de ánimo se vea demasiado afectado. En este hecho reside la clave para pensar que el dolor no significa necesariamente sufrimiento y que, por lo tanto, podemos aprender de las personas que logran sobrellevar el dolor, para enseñar sus formas de afrontarlo a aquéllas que el dolor no las deja vivir.

Esto es, debemos distinguir entre las variables "dolor" y "discapacidad", porque no siempre van unidas. En la mayoría de estudios sobre dolor crónico desde una perspectiva psicológica se utiliza como variable dependiente la "discapacidad". Esto es, se intenta averiguar más qué factores afectan a la discapacidad que cuáles inciden en el propio dolor. La discapacidad, como ocurre con la mayoría de variables, no es definida o evaluada de forma idéntica por los diferentes estudiosos del tema. Con propósitos estrictamente didácticos, en este artículo consideraremos la discapacidad como el grado en que las emociones, las diferentes áreas de la vida (laboral, sexual, sueño, tiempo libre,...) y algunos procesos orgánicos se ven afectados por el dolor. Por lo tanto, para nosotros, mayor discapacidad significa menor calidad de vida a nivel físico, psicológico y social. La variable discapacidad sería, como vemos, una variable que englobaría muchos aspectos distintos entre sí. Consistiría en una especie de abstracción que realizamos para esquematizar la complejidad de los factores que intervienen en el proceso doloroso.

Englobar distintos factores dentro de la variable discapacidad nos permite elaborar un esquema más sencillo. No obstante, este agrupamiento en una misma variable presenta un inconveniente y es que no nos permite hablar o estudiar las relaciones que existen entre los factores que enmarcamos dentro 
del término "discapacidad". Esto es, no nos permite analizar, por ejemplo, las relaciones que hay entre la depresión y el sueño, o la depresión y la ansiedad $\mathrm{y}$, en definitiva, entre cualquiera de las variables que introducimos en el mismo paquete. Por lo tanto, somos conscientes de que nuestro esquema facilitará una comprensión global del proceso doloroso pero que para realizar exámenes más detallados se deberían considerar por separado todas las variables que encajamos dentro del término "discapacidad".

La primera pregunta que nos formulamos respecto a la discapacidad es: ¿por qué personas que sufren la misma patología tienen diferentes grados de discapacidad?

\section{¿Qué factores determinan la discapacidad?}

Podríamos pensar que la discapacidad depende del grado de "daño tisular", sin embargo, aunque efectivamente la causa orgánica puede constituir un determinante de la discapacidad, no es la única. Si pensáramos en estos términos (daño tisular = discapacidad), sería como dar un paso atrás y volver a las antiguas definiciones de dolor donde existía una clara proporcionalidad entre la agresión tisular y la sensación de dolor. Las investigaciones a este respecto apuntan que los factores cognitivos y conductuales son también claros determinantes de la discapacidad. Cómo afecta el padecimiento del dolor a las diferentes áreas de la vida depende de las conductas y pensamientos de los pacientes. ¿Cuáles son las conductas y los pensamientos que producen más discapacidad?

Con el fin de facilitar el análisis de los factores psicológicos que determinan la discapacidad, sería útil contar con la ayuda de un modelo teórico que nos guiara. Si miramos hacia atrás, desde los años 60, en los que se consideraba el dolor como una experiencia sensorial, han ido apareciendo diferentes teorías del dolor. La primera que rompió con los esquemas de aquellos años fue la teoría de la puerta del control de Melzack y Wall (1965). Desde entonces, los modelos han ido evolucionando; en un principio, y como es lógico teniendo en cuenta la historia de la psicología académica, las teorías eran básicamente conductuales (Fordyce, Fowler y De Lateur, 1968) y poco a poco han ido incorporando conceptos cognitivos y emocionales. Una buena revisión sobre la evolución de estos modelos se puede encontrar en el artículo de Sharp (2001). Este autor, después de una revisión y crítica acerca de los principales modelos, propone un nuevo modelo que, según él, es una reformulación de los modelos cognitivos-conductuales ya existentes en el campo de la ansiedad. Sin embargo, creemos que para el estudio del dolor no es necesario proponer una nueva teoría sino que el modelo de Lazarus (Lazarus y Folkman, 1986), tan primordial en el campo del estrés, puede ser traducido a la experiencia del dolor. De hecho, la nueva reformulación de Sharp tiene multitud de puntos en común con la teoría de Lazarus y no aporta ningún concepto totalmente nuevo. Además, muchas investigaciones en el campo del dolor ya se vienen basando en la teoría de Lazarus y por ello no creemos conveniente cambiar hacia una propuesta que no aporta conceptos nuevos (véase la descripción de la teoría de Lazarus 
aplicada al campo del dolor en el libro de Esteve y Ramírez, 2003). Sin embargo, aunque muchos trabajos dicen partir del modelo de Lazarus y manejan conceptos contemplados en dicha teoría, no se identifica explícitamente a qué variables del esquema de Lazarus se hace referencia.

Jensen, Turner, Romano y Karoly realizaron en 1991 una revisión sobre el campo del dolor desde la perspectiva del modelo de Lazarus. Esto es, intentaron poner en orden las evidencias que aportaban distintas investigaciones utilizando el modelo de Lazarus como herramienta. Siguiendo en la tarea de ordenar las evidencias de las que disponemos, nuestro objetivo consistirá en revisar las investigaciones más recientes en esta área integrando en dicho modelo los conceptos que aparecen para contribuir a la consolidación del mismo como una teoría que nos pueda ser útil tanto a nivel teórico como práctico.

Resumiendo el modelo de Lazarus diremos que las situaciones generan más o menos estrés según cómo las personas las evalúen y cómo las afronten. Cuanto más amenazante se evalúe un evento y menos efectivas sean las estrategias de afrontamiento empleadas, mayor será el grado de estrés. Traduciendo este modelo al proceso doloroso, podríamos hipotetizar que el dolor provocará más o menos discapacidad según cómo se evalúe y cómo se afronte. Por lo tanto, deberíamos analizar dos variables para entender los factores determinantes de la discapacidad: la evaluación cognitiva y las estrategias de afrontamiento.

\section{Evaluación cognitiva}

La evaluación cognitiva se refiere a cómo la persona percibe su situación: si evalúa el dolor como algo controlable o no, si cree que tiene habilidades para afrontar su dolencia o no...

En la literatura sobre dolor crónico nos encontramos con una variable muy estudiada que consideramos que encaja perfectamente en el concepto de "evaluación cognitiva". Se trata de la variable "catastrofismo". Los individuos que tienen una visión catastrofista de su dolor son aquéllos que evalúan su dolor como incontrolable, exageran las propiedades amenazantes del estímulo doloroso y no pueden apartar su mente del dolor (Sullivan, Bishop y Pivik, 1995; Thorn, Bothby y Sullivan, 2002; Turner, Jensen y Romano, 2000). Existe una gran polémica sobre si esta variable debe considerarse una estrategia de afrontamiento, o un tipo de creencia o evaluación. Sin entrar en este debate, sólo queríamos indicar que, según nuestra opinión, es mejor no considerarla como estrategia de afrontamiento puesto que no encaja totalmente con la definición de Lazarus, dado que como veremos más adelante las estrategias se definen como esfuerzos cognitivos y conductuales. Por lo tanto, los procesos que no requieren esfuerzo no se pueden clasificar como estrategias (Moix, 1990). Y nuestra experiencia nos indica que se trata más de una evaluación de tipo automático que no requiere esfuerzo alguno.

En las investigaciones en las que se evalúa esta variable, se puede comprobar la clara asociación que existe entre catastrofismo y discapacidad. Esto es, cuanto mayor es el catastrofismo mayor es la discapacidad, e incluso mayor es la intensidad del dolor (Bishop, Ferraro, Borowiak, 2001; Buer y Linton, 
2002; Severeijns, Vlaeyen, Van Den Hout y Weber, 2001; Tan, Jensen, Robinson, Thornby y Monga, 2001; Turner, Jensen, Warms y Cardenas, 2002; Van-den-hout, Vlaeyen, Heuts, Sillen y Willen, 2001). En términos generales, el dolor resulta más incapacitante en las personas que presentan un mayor número de pensamientos catastróficos, es decir, las diferentes áreas de su vida resultan más alteradas por culpa del dolor y sufren más depresión y ansiedad. En un estudio donde 88 mujeres con dolor crónico debían anotar durante 30 días en un diario sus pensamientos catastróficos, el nivel de dolor y el estado de ánimo, se pudo comprobar que los días en los que se registraban más pensamientos catastróficos, incrementaba el dolor y los estados de ánimo negativos (Grant, Long y Willms, 2002).

Si ahondamos más en la variable catastrofismo, podemos ver que este tipo de pensamiento no sólo afecta a los estados de ánimo sino también a otros procesos cognitivos como los atencionales. Crombez (2002) pudo comprobar en dos estudios experimentales que las personas con más pensamientos catastrofistas eran las que, cuando tenían que llevar a cabo una tarea en la que se evaluaba su tiempo de reacción, su nivel atencional se encontraba más interrumpido por el dolor que el de aquellos sujetos con menos pensamientos catastróficos. Podríamos concluir que la disminución en la capacidad de concentración que presentan, en muchos casos, los pacientes con dolor crónico está mediada por la variable catastrofismo. Grisart y Vanderlinden (2001) pudieron comprobar cómo el catastrofísmo no sólo altera los procesos atencionales sino que también afecta negativamente a la memoria.

Parece que los pensamientos catastróficos tienen efectos más devastadores a medida que pasa el tiempo. En un trabajo donde se evaluó el efecto del pensamiento catastrofista sobre la discapacidad en tres grupos de pacientes en los que la duración de su patología era diferente, se pudo comprobar que en aquellos sujetos en los que su patología se remontaba a más tiempo atrás, los pensamientos catastróficos incidían en mayor medida en su discapacidad. (Sullivan, Sullivan y Adams, 2002).

Una clara conclusión aplicada que podemos extraer sobre los resultados de los estudios acerca del catastrofismo, se refiere a la necesidad de intentar disminuir este tipo de pensamientos, para poder así reducir también la discapacidad. De hecho, Thorn, Boothby y Sullivan (2002) han elaborado un programa muy detallado con este fin.

Dentro de la variable "evaluación cognitiva" también podemos encontrar otros aspectos sobre los cuales se puede evaluar el proceso doloroso.

Como ya hemos indicado, dentro de la "evaluación cognitiva" se encuentra la valoración que la persona realiza sobre sí misma. A este respecto diversas investigaciones ponen de relieve que los pacientes que se consideran con menor competencia para solucionar problemas son los que sufren más dolor, más depresión e inadaptación (Kerns, Rosenberg y Otis, 2002; Witty, Heppner, Bernard y Thoreson, 2001).

$\mathrm{La}$ autoeficacia es un concepto claramente ligado al anterior dado que se refiere a lo capaz que el individuo se siente de solucionar o afrontar una situación concreta (sea o no el dolor). En el caso de pacientes con dolor, hace referencia 
a lo capaces que se sienten de manejar el proceso doloroso. En general, los estudios que evalúan esta variable comprueban que las personas más autoeficaces son las que sienten menos dolor, manejan mejor su dolor y otros síntomas, muestran una menor discapacidad (menos depresión y ansiedad) y mejores resultados en los tratamientos (Martín-Aragón et al., 1999; Pastor, MartínAragón, M. y Lledó, 1999; Strong, Westbury, Smith, Mckenzie y Ryan, 2002; Turk y Okifuji, 2002). Tal como apuntan Turk y Okifuji (2002), probablemente, la autoeficacia afecta al dolor y a la adaptación a través de dos vías: por una lado, porque probablemente aquellos sujetos con mayor autoeficacia deben movilizar más recursos y estrategias para afrontar su problema y, por otro, los pacientes que se sienten más autoeficaces, piensan que su dolor es más controlable y experimentan menos ansiedad.

Resumiendo este apartado, podemos concluir que la evaluación cognitiva (concretamente: el catastrofismo, las habilidades subjetivas para solucionar problemas y la autoeficacia) incide claramente en la discapacidad e incluso en el propio dolor. En el modelo de Lazarus, como ya hemos apuntado, la ansiedad, en este caso el dolor, no sólo depende de la evaluación cognitiva, sino también de cómo el sujeto afronta la situación. Vamos a analizar seguidamente esta variable.

\section{Estrategias de afrontamiento}

Las estrategias de afrontamiento se definen como los esfuerzos cognitivos y conductuales para superar la situación potencialmente amenazante, en el caso que nos ocupa, el dolor.

Los estudios que intentan relacionar las estrategias de afrontamiento y la discapacidad suelen mostrar que las estrategias activas y dirigidas a la solución de problemas parecen estar relacionadas con una menor discapacidad e incluso menor dolor, mientras otras estrategias más pasivas están asociadas a un mayor nivel de dolor (Esteve, López y Ramírez, 1999; Grant, Long y Willms, 2002; McCraken, Goetsch y Semenchuk, 1998; Rodríguez-Parra, Esteve y Martínez, 2000). Estrategias concretas que podríamos calificar como pasivas son las fantasías esperanzadoras (por ejemplo, imaginarse que el dolor terminará pronto) y el rezo.

Respecto a la distracción como estrategia de afrontamiento, los resultados parecen ser contradictorios, mientras algunos estudios indican que se trata de una estrategia adaptativa, otros muestran lo contrario (Grant, Long y Willms, 2002; Rodríguez-Parra, Esteve y López, 2000).

La "expresión de las emociones" también la podemos considerar una forma de afrontamiento. Keefe y colaboradores, después de una exhaustiva revisión sobre la expresividad emocional, llegan a la conclusión de que la no expresión de emociones está asociada a un mayor dolor (Keefe, Lumley, Anderson, Lynch y Carson, 2001). En este sentido, una de las emociones más estudiadas es la ira, habiéndose observado que la inhibición de su expresión está correlacionada tanto con el dolor como con la discapacidad (Casado y Urbano, 2001). 
La "búsqueda de apoyo social", en cierto modo relacionada con la estrategia comentada anteriormente, también parece constituir una estrategia adaptativa. Como apuntan Comeche y Lasa (2001), los trabajos al respecto indican una relación negativa entre la utilización de esta estrategia y el nivel de dolor.

"La toma de medicación" podríamos considerarla asimismo como una estrategia de afrontamiento. A este respecto, podemos encontrar pacientes que se medican en exceso y otros que, por miedo a los efectos secundarios, no siguen las prescripciones médicas. Los efectos iatrogénicos de una inadecuada medicación pueden dar lugar a alteraciones fisiológicas (Sharp, 2001). Por lo tanto, la toma de medicación es un ejemplo más de cómo las estrategias de afrontamiento inadecuadas pueden provocar discapacidad.

Aunque todavía necesitamos más estudios para clarificar qué estrategias pueden resultar beneficiosas o perjudiciales para afrontar el dolor, por el momento sí podemos afirmar que las estrategías parecen influir sobre la discapacidad.

\section{Variables predictoras indirectas}

Además de la evaluación cognitiva y de las estrategias de afrontamiento, en la literatura sobre el dolor crónico nos encontramos con otras variables que también parecen predecir, en mayor o menor medida, el dolor y/o la discapacidad. Estas variables son factores que en el modelo de ansiedad de Lazarus también aparecen y que son contempladas como variables de segundo orden ya que afectan a la ansiedad, pero siempre a través de la evaluación cognitiva y las estrategias de afrontamiento. En el caso del dolor, parece que ocurre lo mismo. Esto es, existen numerosas variables que inciden en el dolor, y posiblemente lo hagan mediante la modulación de la evaluación cognitiva y las estrategias de afrontamiento. Este apartado estará dedicado a la descripción de las mismas.

\section{Creencias}

En los modelos cognitivos, las creencias siempre juegan un papel crucial y, cómo no, en el modelo de Lazarus también. Las creencias son nociones preexistentes o esquemas cognitivos de la realidad que moldean o transforman nuestras percepciones de lo que nos envuelve y de nosotros mismos (véase Camacho y Anarte, 2001). Éstas se pueden referir a múltiples aspectos. Creencias sobre el mundo en general, sobre la salud, sobre uno mismo, ...y sobre el dolor (acerca de la etiología, diagnóstico, tratamiento, duración del dolor, etc.).

Como el dolor es un fenómeno tan amplio que integra aspectos físicos, psicológicos y sociales, las creencias que pueden afectarlo, más o menos directamente, son innumerables. A continuación sólo expondremos las que hemos encontrado descritas en la literatura.

Las creencias sobre los factores que afectan al dolor pueden determinar la forma de afrontarlo. Por ejemplo, a los pacientes que crean que la actividad agrava el daño tisular, esta idea puede conducirlos a la inactividad, la cual por 
sí misma puede agravar el problema; esto es, aumentar la discapacidad y el dolor (Buer y Linton, 2002; Turk y Okifugi, 2002).

Las creencias sobre la tipología del dolor también pueden afectar al tipo de tratamiento que prefiere el paciente. Aquellos pacientes que consideran su dolor como un trastorno totalmente físico suelen preferir terapias más médicas y no suelen aceptar participar en programas interdisciplinares o psicológicos, y si lo hacen suelen abandonar o mostrar poca adherencia (Turk y Okifugi, 2002). Este tipo de creencias puede afectar a más aspectos. En un estudio de Walsh y Radcliffe (2002), se pudo comprobar que los pacientes que evaluaban su dolor como un trastorno muy "orgánico" fueron los que presentaron un mayor índice de discapacidad. Más aún, cuando los pacientes fueron tratados mediante un programa interdisciplinar, se comprobó que aquéllos en los que se redujo en mayor medida la visión del dolor como un fenómeno puramente orgánico, fueron los que presentaron mayor mejoría.

El locus de control puede afectar asimismo la discapacidad. Parece que los pacientes con un locus de control interno, a saber, aquéllos que creen que el dolor depende de ellos mismos son los que se adaptan mejor a su situación, si los comparamos con personas con locus de control externo que consideran que su dolor depende de otros factores (Camacho y Anarte, 2001).

\section{Rasgos de personalidad o psicopatologías}

Existe una línea de investigación que intenta encontrar relaciones entre rasgos de personalidad o patologías de personalidad, y el padecimiento de dolor crónico y la discapacidad. Su objetivo es encontrar si existen pacientes con tipos de personalidad o psicopatologías que tengan más tendencia a padecer dolor crónico o a sentirse más incapacitados. En un reciente estudio llevado a cabo por Mayr, Rose y Johstone (2002) en el que entrevistaron a 245 pacientes que sufrían dolor lumbar, se encontró que el porcentaje de pacientes que sufría alguna psicopatología era menor entre los pacientes en los que se había detectado una clara causa orgánica de su dolor, que entre aquéllos en que no se había detectado causa alguna.

Las psicopatologías más relacionadas con el dolor crónico son la depresión y la ansiedad (Dersh, Polatin, Gatchel, 2002; Mayr, Hogler, Ghedina y Berek, 2003), aunque no está claro en qué dirección va la influencia (Fishbain, 2002). Tal como apuntan Serrano y colaboradores, existen numerosos autores que plantean serias dudas acerca de que el dolor pueda ser atribuido a una personalidad neurótica premórbida (Serrano, Cañas, Serrano, García y Caballero, 2002). La revisión realizada por estos autores parece indicar más bien que la personalidad premórbida, aunque no sea la precursora del dolor, determina en parte la discapacidad o sufrimiento provocado por éste. Dersh, Polatin y Gatchel (2002), después de una extensa revisión de la literatura sobre este tema, concluyen, de forma similar a Serrano y colaboradores (Serrano et al., 2002), que se podría considerar a las psicopatologías como características preexistentes en stand by que son activadas por el estrés que supone el sufrimiento de dolor 
crónico. Así, aunque quizás las psicopatologías no son las responsables del inicio del dolor, probablemente intervengan en su cronificación.

Intentando encajar esta idea en el modelo teórico que nos está guiando, probablemente los rasgos o patologías de personalidad afecten a la discapacidad a través de su influencia en la evaluación cognitiva y las estrategias de afrontamiento. A este respecto, por ejemplo, se ha comprobado que la relación existente entre neuroticismo (rasgo de personalidad) y dolor crónico parece estar mediada por el catastrofismo (evaluación cognitiva) (Ramírez, 2002; Ramírez, Esteve y López, 2001). Esto es, los pacientes con niveles mayores de neuroticismo padecen mayor dolor porque son los que más pensamientos catastróficos presentan.

\section{Historia previa}

En cualquier trastorno, la historia previa del paciente es una variable que puede incidir tanto en el inicio, como en el mantenimiento o curación del mismo. El dolor crónico no es una excepción. La historia previa es una variable casi inabarcable ya que incluye el devenir del paciente desde su nacimiento. Por lo tanto, incluye una infinidad de aspectos.

En el caso del dolor crónico varios de estos aspectos se han mostrado determinantes. Uno de ellos es el sufrimiento de dolor crónico por parte de los progenitores. Parece que cuando se comparan pacientes con dolor crónico con sujetos controles, los primeros tienen mayor probabilidad de tener padres que también sufren dolor crónico.

Otro aspecto que también parece incidir en el padecimiento de dolor crónico es la historia de abusos sexuales. Green y colaboradores en un estudio donde se entrevistó a 90 mujeres con dolor crónico, pudieron comprobar que aquéllas que presentaban una historia más larga de abusos sexuales eran las que sufrían mayor ansiedad y más dolor (Green, Flowe-Valencia, Rosenblum y Tait, 2001). En la misma línea, Goldberg y Goldstein, comparando un grupo de pacientes con dolor crónico con un grupo control, constataron que un 54\% de los pacientes con dolor crónico manifestaban haber vivido abusos sexuales o verbales en su niñez, mientras en el grupo control este porcentaje se reducía al $21.4 \%$. Aunque en la literatura podemos encontrar más trabajos que corroboran los resultados anteriores (Finestone, et al., 2000), según McBeth no deberíamos fiarnos totalmente de este tipo de estudios ya que al ser retrospectivos, el hecho de que los pacientes con dolor manifiesten haber padecido más abusos, no nos indica con certeza que realmente los hayan sufrido, sino solamente muestra que recuerdan haberlos sufrido (McBeth, Morris, Benjamin, Silman y MacFarlane, 2001). Dado que la memoria de este tipo de pacientes puede estar sesgada hacia lo negativo, probablemente las informaciones obtenidas a través de entrevistas no son comparables con informaciones extraídas de entrevistas a personas sin dolor y por lo tanto sin este tipo de sesgo.

En general, parece que no sólo los abusos sexuales, sino cualquier tipo de evento estresante puede incidir en el dolor. Lampe y colaboradores, al comparar dos grupos de pacientes con dolor crónico de espalda (un grupo con causa orgánica y el segundo con causa idiopática), observaron que en los pacientes 
cuyo dolor se debía a causas idiopáticas había un mayor número de eventos antes de la exacerbación de su dolor (Lampe et al., 1998). La relación entre eventos estresantes y dolor se ha podido comprobar en diferentes investigaciones (Craufurd, Creed y Jayson, 1990; De Benedittis, Lorenzetti y Pieri, 1990).

\section{Apoyo social}

El apoyo social es una variable determinante de la ansiedad tal y como se contempla en el modelo de Lazarus. En el caso del dolor, el apoyo social también se muestra como un factor modulador. Concretamente, parece que aquellos pacientes que indican tener un mayor apoyo social son los que sufren menos dolor y discapacidad (Kerns, Rosenberg y Otis, 2002). El apoyo social puede ayudar a los pacientes a través de dos vías: emocional y práctica. Es probable que los pacientes con mayor apoyo social tengan más oportunidades de expresar sus sentimientos en torno al dolor y puedan obtener más información y ayuda que les facilite solventar las situaciones problemáticas que se presenten. En otras palabras, el apoyo social incidirá sobre el dolor y la discapacidad a través de las estrategias de afrontamiento.

\section{Nivel educacional}

Roth y Geisser (2002), en un estudio en el que se evaluaron 299 pacientes con dolor crónico, pudieron comprobar que a menor nivel educacional más inadaptación a causa del dolor. La relación entre estas dos variables, según esta investigación, parece estar mediada por el catastrofismo y la creencia de que el dolor es la señal de daño tisular ya que las personas con un nivel educacional más bajo presentan más pensamientos catastróficos y una mayor tendencia a creer que el dolor señaliza daño tisular. Así pues, se trata de una variable indirecta que afecta a la discapacidad a través de la evaluación cognitiva (concretamente el catastrofismo).

Resumiendo, en este apartado hemos podido comprobar cómo las formas de evaluar y afrontar el dolor determinan, en gran parte, la discapacidad que éste provocará en la vida cotidiana. A su vez, la forma de evaluar el dolor y de afrontarlo vendrá determinada por multitud de factores indirectos (creencias, rasgos de personalidad, historia previa, apoyo social y nivel educacional). En la figura 1 de la página siguiente, podemos encontrar un esquema que refleja estas influencias. Las influencias de este esquema se ponen en marcha al iniciarse la sensación dolorosa, la cual conlleva que se dé una evaluación de la misma y que se empleen unas estrategias para afrontarla. Debemos tener precaución al interpretar el esquema presentado, puesto que aunque las flechas sugieren influencias de tipo causal, los estudios en los que nos basamos, al ser la mayoría de tipo correlacional, no nos permiten asegurar esta causalidad. Por lo tanto, el modelo propuesto se debe considerar hipotético.

Si nos fijamos detenidamente en la figura 1, podemos comprobar que la discapacidad provocada por el dolor, de forma más bien indirecta a través de la 


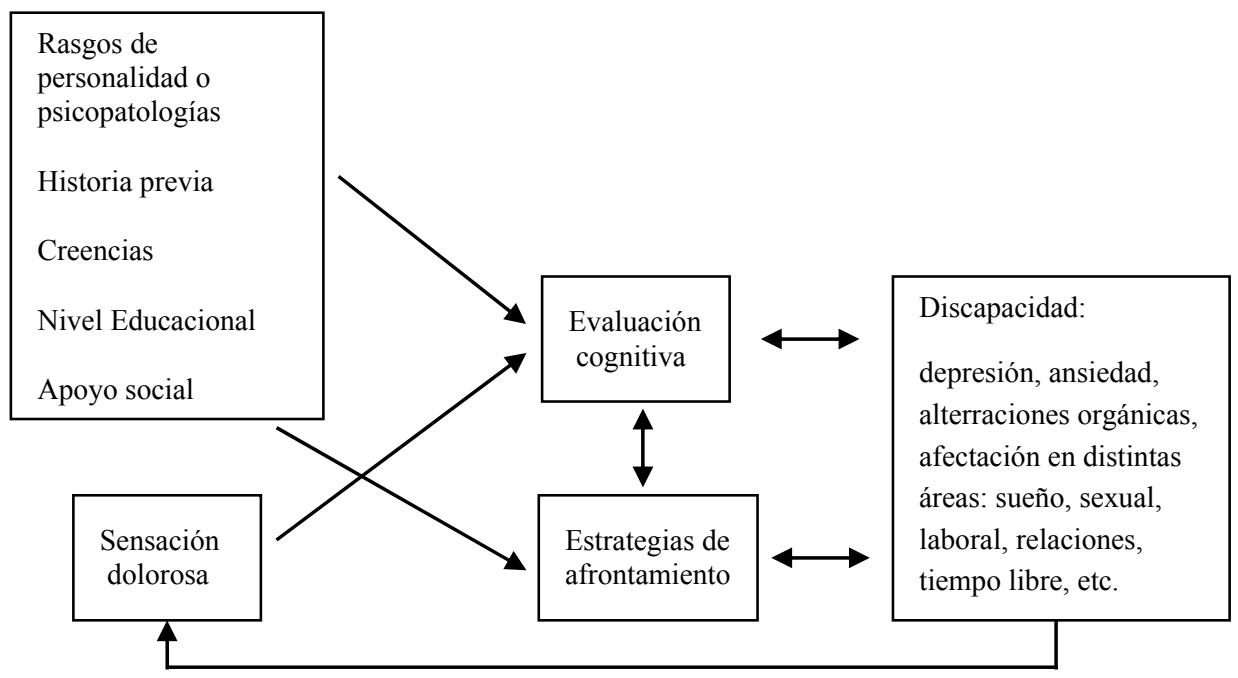

Figura 1. Factores psicológicos predictores de la discapacidad y la cronicidad.

evaluación y las estrategias, a su vez afecta al mismo. Esto es, se presenta un círculo dolor - discapacidad - dolor. Este círculo explicaría, en parte, la cronicidad del dolor. La pregunta que nos podríamos formular es la que desarrollamos a continuación.

\section{¿Cuáles son los factores psicológicos predictores de la cronicidad?}

En muchos casos nos podemos encontrar pacientes con dolor crónico en los que su dolor es "desproporcionado" en relación con su causa orgánica. En otros casos, incluso nos podemos encontrar dolor crónico cuando el motivo desencadenante inicial ha desaparecido completamente. ¿Cómo podemos explicar la cronicidad, la continuación de un dolor sin causa o con una causa orgánica que no es proporcional al grado de dolor?

Como ya hemos comentado en la figura 1, podemos encontrar una flecha que parte de la discapacidad y se dirige a la sensación dolorosa, cerrando de esta forma un círculo. ¿Cómo podemos explicar esta flecha; esto es, la influencia de la discapacidad sobre el dolor? Existen varias hipótesis. Estas explicaciones o hipótesis no se enmarcan en la teoría de Lazarus, porque no olvidemos que es una teoría sobre estrés y no sobre dolor y, por lo tanto, no da respuesta a vías concretas de cronificación del dolor. Sin embargo, las hipótesis que vamos a exponer a continuación no se contradicen con el modelo de Lazarus, sino que serían explicaciones a otro nivel más detallado de la influencia de la discapacidad sobre el dolor. A continuación vamos a comentar las hipótesis más extendidas. 
Uno de los mecanismos, desde un punto de vista más fisiológico, que se han argumentado para explicar la cronicidad del dolor consistiría en la sensibilización de las vías de trasmisión. Las neuronas son plásticas y sufren cambios debidos a las experiencias. Gracias a esta plasticidad aprendemos, pero desgraciadamente este mismo valioso mecanismo puede ser el responsable de la cronificación del dolor puesto que, aunque la causa inicial del dolor desaparezca, las vías han sufrido cambios, se han "sensibilizado", y siguen trasmitiendo dolor (Sufka, 2000).

Según Brosschot (2002), existen dos tipos de sensibilización: una más física que residiría en las vías de trasmisión del dolor (ya enumerada) y otra de tipo más psicológico. Esta última la podríamos considerar como un posible círculo vicioso: dolor - ansiedad - hipervigilancia - dolor. Esto es, la ansiedad o preocupación que provoca el dolor aumenta la alerta o la atención que las personas prestan a las sensaciones dolorosas (Roelofs, Peters, Zeegers y Vlaeyen, 2002) aumentando la sensación subjetiva de dolor (Jansen, 2002). De hecho, parece que las personas con dolor crónico no solamente presentan sesgos atencionales, sino también de interpretación y memoria. Esto es, comparados con la población normal tienen tendencia a interpretar o percibir estímulos físicos como dolorosos y a recordar en mayor medida los episodios con dolor (Pincus y Morley, 2001.)

Otra posible hipótesis describe el dolor como impulsor de un círculo de dolor - ansiedad - tensión - dolor. Así, aunque desaparezca el desencadenante inicial la rueda ya está en marcha (Jansen, 2002). De hecho, cada vez son más numerosas las investigaciones que muestran que aquellos pacientes que experimentan más ansiedad son los que sufren más dolor (Riley, Robinson, Wade, Myers y Price, 2001; Serrano, Cañas, Serrano, García y Caballero, 2002).

También podemos encontrar explicaciones a nivel conductual. En estas hipótesis, las conductas de dolor se refuerzan por distintos acontecimientos (atención de los allegados, bajas laborales, etc.) que mantienen el dolor. Romano y colaboradores llevaron a cabo un estudio en el que grabaron a 121 pacientes con dolor crónico realizando algunas tareas del hogar con sus parejas, mostrando cómo los comportamientos solícitos (ayudar en exceso, no dejar hacer algunas actividades...) por parte de la pareja aumentaban las conductas de dolor (verbales y no verbales) de los pacientes (Romano, Jensen, Turner, Good y Hops, 2000). Incluso algunos estudios de tipo experimental sugieren que las personas con dolor crónico son más fácilmente condicionables instrumentalmente que las personas sin dolor (Flor, Knost y Birbaumer, 2002).

Dentro de las explicaciones conductuales, también se encuentra la hipótesis «miedo al dolor = conductas de evitación» (Jansen, 2002). El miedo a experimentar dolor deriva en conductas de evitación del dolor, concretamente en disminuir, en alto grado, la actividad física (Turk y Okifuji, 2002). La inactividad provocada por el miedo, a su vez, conlleva una debilitación de la musculatura en primer lugar y la alteración de otros procesos fisiológicos que no permitirán la recuperación del paciente y la consiguiente disminución del dolor.

Asimismo, se postulan tesis a nivel cognitivo, proponiendo que el dolor físico puede suponer para algunos pacientes la atribución "perfecta" para su 
depresión; es decir, "la excusa" para su estado de ánimo. De esta forma, la depresión podría cronificar el dolor, pero también lo podría hacer por otras vías más conductuales o fisiológicas. De hecho, la depresión es la emoción más asociada al dolor, existen numerosos estudios que muestran cómo las personas con más depresión son las que sienten más dolor (Reeves, 2000; Serrano et al., 2002).

Dentro de las explicaciones desde un punto de vista más teórico, Esteve, Ramírez y López (2001) basándose en el modelo de procesamiento de las emociones (2001) discuten una nueva idea que aparece en la literatura sobre el dolor que se basa en conceptualizar el dolor como un esquema. Esta hipótesis sugiere que cuando se experimenta en repetidas ocasiones dolor paralelamente con otras emociones (tristeza, miedo, disgusto o ira), se construye un esquema en el que se representan al mismo tiempo las cualidades sensoriales y emocionales de la experiencia del dolor. Esto es, el esquema de dolor que se almacena en la memoria contendría una representación de los estímulos nocioceptivos junto con las emociones, sensaciones e imágenes previamente asociadas. De esta forma, podríamos explicar cómo casi de forma automática la experiencia sensorial del dolor puede provocar malestar emocional dado que se activarían los nódulos emocionales y también, a la inversa, cómo las emociones negativas pueden facilitar la sensación dolorosa.

Las explicaciones expuestas no se contradicen entre sí ya que son hipótesis a diferentes niveles. Parece, pues, que el mantenimiento del dolor es multicausal, y que probablemente, para cada paciente, cada uno de los mecanismos expuestos tendrá "un peso" diferente para explicar su caso. Como vemos, la cronicidad está fuertemente marcada por factores psicológicos y no sólo a nivel de hipótesis, sino que se ha comprobado que los factores psicológicos pueden contribuir a la cronicidad tanto o más que los factores físicos, entendiendo como factores físicos la gravedad del daño tisular (Turk y Okifuji, 2002).

Si como vamos viendo a lo largo de este artículo, el dolor crónico es un fenómeno extremadamente complejo, en el que intervienen factores físicos, psicológicos y sociales, nos podríamos preguntar lo siguiente.

\section{¿Se emplean técnicas auténticamente interdisciplinares para tratar el dolor? ¿son efectivas?}

El tratamiento del dolor crónico ha ido evolucionando, al igual que su definición. Mientras en un principio el dolor se contemplaba desde una perspectiva muy fisiológica, el tratamiento se reducía a un tipo de técnicas médicas (fármacos, intervenciones quirúrgicas, etc.). Sin embargo, a medida que el dolor se define como una afectación más global, la forma de tratarlo también se amplía.

Cada día son más numerosas las publicaciones que concluyen que los tratamientos interdisciplinares del dolor crónico son efectivos (Claiborne, 2002; Collado et al., 2001; Esteban 1999). Los tratamientos psicosociales combinados con los médicos dan mejores resultados que cada uno por separado, 
como muy bien indica Turk: $1+1=3$ (Turk, 2001a). Los tratamientos interdisciplinares no sólo se han mostrado efectivos, sino incluso rentables: pensemos en la reducción que conllevan de bajas laborales, medicación, visitas médicas, etc. (Cipher, Fernández y Clifford, 2001; Turk, 2001b).

No existe un único protocolo de tratamiento interdiciplinar que se emplee de forma estandarizada (Ashburn y Staats, 1999; Biurrun y Jusué, 1998; Górriz, Garrido y Andueza, 1999; Moix, 2003). Acabamos de realizar un análisis del proceso doloroso según el modelo de Lazarus, y aunque resulta un modelo muy útil en el plano teórico y no nos cabe duda de que podría resultar muy útil para guiar el diseño de terapias, no es frecuente que se parta del mismo como guía terapéutica. Habitualmente, las terapias se diseñan guiadas por motivos más pragmáticos. No obstante, muchas de las técnicas utilizadas se pueden analizar desde el modelo de Lazarus como formas de reestructurar la evaluación cognitiva o enseñar formas de afrontamiento más útiles.

Como hemos comentado no existe un único protocolo, sin embargo, si analizamos distintos tratamientos utilizados podemos realizar una abstracción donde se contemplen las características más comunes.

La mayoría de tratamientos interdisciplinares suelen poseer un filosofía educativa. Su objetivo no es tanto limitarse a dar prescripciones sino más bien trata de educar a los pacientes para que ellos sepan afrontar de una forma óptima su dolor.

Muchos de estos tratamientos son grupales. Esto significa que las diferentes sesiones de las que consta el programa no se llevan a cabo de forma individual con un solo paciente, sino que las sesiones se realizan en grupos.

A continuación enumeraremos los profesionales que suelen guiar las sesiones en los programas interdisciplinares y la información que puede aportar cada uno de ellos, deteniéndonos en explicar con más detalle las técnicas tratadas por los psicólogos.

Médicos. Información acerca de las patologías que pueden causar el dolor y de los tratamientos médicos.

Anestesistas. Información sobre técnicas médicas para el tratamiento del dolor. Indicaciones, interacciones y efectos secundarios de los analgésicos más empleados.

Trabajadores sociales. Información legal sobre las bajas laborales. Información sobre recursos para acceder a actividades lúdicas en la localidad del paciente.

Fisioterapeutas. Información sobre higiene postural. Indicaciones sobre ejercicios recomendados.

Psicólogos. A continuación enumeraremos distintas técnicas cognitivoconductuales que suelen emplearse en pacientes con dolor crónico. Estas técnicas se emplean en algunas ocasiones como técnicas aisladas y en otras formando parte de un tratamiento interdisciplinar.

- Conceptualización. Antes de utilizar las distintas técnicas cognitivoconductuales, se suele presentar una explicación teórica sencilla de cómo los factores psicológicos actúan sobre el dolor y, por lo tanto, de la necesidad de utilizar técnicas psicológicas. 
- Técnicas de relajación. Sumamente útiles para romper el círculo dolor tensión - dolor. La técnica de relajación más empleada es la de Jacobson, aunque las técnicas de biofeedback también se han mostrado eficaces (Mas y Comeche, 2001; Middaugh y Pawlink, 2002).

- Técnicas de hipnosis. En algunos casos, además de emplearse técnicas de relajación se puede utilizar la hipnosis. La eficacia de la hipnosis en el tratamiento del dolor se ha probado en numerosas investigaciones (Gay, 2002, Hawkins, 2001). Una detallada descripción de esta técnica se puede encontrar en Moix (2002).

- Reestructuración cognitiva. Si como hemos comentado anteriormente la discapacidad que provoca el dolor depende de cómo se evalúe, es lógico que el objetivo principal de la reestructuración cognitiva consista en cambiar la forma en que los pacientes evalúan el dolor. Más concretamente se trataría de modificar los pensamientos catastrofistas y de fomentar la creencia del dolor como algo controlable (Lebovits, 2002; Thorn, Bothby y Sullivan, 2002).

- Entrenamiento en asertividad. El dolor suele acrecentar los problemas de relación con los demás (familiares, profesionales de la salud, etc.) y, por lo tanto, es conveniente enseñar técnicas para intentar mejorar dichas relaciones.

- Condicionamiento instrumental para disminuir conductas de evitación. El miedo al dolor provoca que disminuya la actividad en muchos pacientes. Con el fin de reducir las conductas de evitación se emplean en muchos casos técnicas basadas en el condicionamiento instrumental, concretamente se pide a los sujetos que realicen progresivamente distintas actividades, reforzando cada uno de los pasos logrados (Díaz-Sibaja, Martín y Díaz-García, 2001; Turk y Okifuji, 2002; Vlaeyen, De Jong, Onghena, Kerkhoffs-Hanssen y Kole-Snijders, 2002).

- Higiene del sueño. En muchos casos, el dolor trae consigo problemas para conciliar o mantener el sueño, por este motivo es importante tratar este punto dando unas pautas que guíen a los pacientes (Currie, Wilson y Curran, 2002).

- Sexualidad. Las relaciones sexuales se ven afectadas por el dolor. Es necesario tratar este punto desde diferentes perspectivas: los fisioterapeutas pueden indicar posturas más convenientes, los anestesistas indicar algunos efectos secundarios de los fármacos que pueden tener repercusión a nivel sexual. Y en el plano más psicológico es importante tratar este punto con más profundidad: el significado del sexo para el paciente, su evolución, etc.

- Aumento de actividades distractoras. Uno de los objetivos consiste en aumentar el tiempo que los pacientes dedican a realizar actividades agradables. En muchos casos, debido a la depresión a la que va asociada el dolor, las actividades agradables se ven claramente reducidas.

- Organización del tiempo. El dolor significa para muchos pacientes no poder llevar el mismo ritmo que antes de sufrirlo, por este motivo en muchas ocasiones se ven desbordados y necesitan una guía para organizar mejor su tiempo.

Este tipo de sesiones no sólo se limitan a dar información; en muchos casos también se realizan ejercicios y se dan "deberes" que los pacientes tienen que realizar en sus casas.

En muchas ocasiones, las sesiones grupales se intercalan con sesiones individuales donde los profesionales pueden tratar y dar información más per- 
sonalizada. En el caso de las sesiones individuales que llevan a cabo los psicólogos, los pacientes tendrán un espacio donde expresar sus emociones y donde tratar problemas más íntimos.

Como ya hemos comentado anteriormente, son muchos los estudios que muestran la efectividad de las técnicas interdisciplinares. Además de este tipo de técnicas, en algunas ocasiones también se emplean las técnicas cognitivoconductuales de forma aislada, sin pertenecer a un programa interdisciplinar más amplio, mostrándose asimismo efectivas (Moore, Von-Korkk, Cherkin, Saunders y Lorig, 2000; Pastor, Pons, Lledó, Martín-Aragón, López-Roig, Terol y Rodríguez-Marín, 2003). Sin embargo, su efectividad aumenta cuando se integran en un programa interdisciplinar (Turk, 2001b).

Desafortunadamente, aunque la efectividad de las técnicas psicológicas o interdisciplinares no se puede poner en duda por las abundantes investigaciones que la avalan, dichos tratamientos presentan algunos problemas que necesitan ser investigados con urgencia.

En muchos casos, los pacientes no aceptan participar en este tipo de programas, a pesar de su demostrada efectividad. El número de casos que abandona el tratamiento también es considerable (Strong, Westbury, Smith, McKenzie y Ryan, 2002). Y la poca o nula adherencia que muestran muchos pacientes al tratamiento también se convierte en un grave problema, ya que los pacientes que no siguen las indicaciones (ejercicios, relajación, nuevas estrategias de afrontamiento, etc.) no se benefician del tratamiento. Hablar de programas eficaces, cuando existen estas incidencias, no favorece la optimización de los programas. Como apuntan Turk y Okifuji (2002), quizás deberíamos considerar que si no conseguimos que los pacientes acepten participar, acaben el tratamiento y sean adherentes, realmente el programa presenta serias deficiencias y etiquetarlo de eficaz es más que dudoso. Por lo tanto, dentro de los objetivos terapéuticos de cualquier programa se deberían añadir: (1) conseguir la participación; (2) evitar los abandonos; y (3) aumentar la adherencia. Lamberg (2002), sugiere muy acertadamente, que los médicos deben asegurarle al paciente que su dolor es orgánico y real, y que no es psicológico y luego recomendarles las técnicas cognitivo-conductuales como complemento de las terapias médicas. Si a los pacientes se les deriva hacia técnicas cognitivoconductuales o programas educativos interdisciplinares sin este tipo de explicación, pueden pensar que el médico está sugiriendo que su dolor es psicológico $\mathrm{y}$, por lo tanto, rehusar este tipo de tratamiento.

Otro aspecto que debería ser investigado respecto a los tratamientos de dolor crónico, se refiere a la posible efectividad de los grupos de autoayuda. En algunas ocasiones, pacientes con dolor crónico de algún tipo (fibromialgias, dolor de espalda, etc.) se agrupan con el fin de ayudarse mutuamente (compartir información, apoyarse psicológicamente, compartir actividades recreativas, etc.). Aunque estos grupos existen, parece que se ha estudiado poco su efectividad. A este respecto, sólo hemos obtenido información de una investigación en la que se muestra cómo aquellos pacientes instruidos sobre autocuidados de la lumbalgia por otros pacientes que padecen la misma dolencia, adoptaron una actitud más positiva y redujeron más rápidamente su discapacidad que los 
atendidos de forma tradicional (Von-Korf et al., 1998). Estos resultados sugieren que quizás las terapias interdisciplinares descritas en el presente estudio aumentarían su efectividad si incluyeran no sólo a especialistas de distintos ámbitos médicos sino a los propios pacientes como personal instructor.

Asimismo, consideramos que el momento de aplicación del tratamiento es un aspecto en el que se debe profundizar. Los tratamientos hasta aquí apuntados se dirigen a pacientes que ya padecen dolor crónico. Sin embargo, podríamos empezar a preguntarnos si también sería eficaz aplicar técnicas para prevenir el dolor. Los estudios sobre la efectividad de técnicas preventivas constituyen una excepción, pero arrojan resultados esperanzadores que indican que a través de técnicas cognitivo-conductuales aplicadas a personas que no son pacientes pero que han tenido algún episodio de dolor de espalda, se pueden prevenir muchas repercusiones del dolor (días de baja laboral, días con dolor, desadaptación, etc.) (Linton y Ryberg, 2001). Incluso se han realizado macrocampañas para informar a la población general sobre la lumbalgia (síntomas, consejos sobre mantener la actividad y no dejar de trabajar, higiene postural, etc.), obteniéndose resultados muy positivos. En un trabajo donde se compararon dos estados: en uno de ellos se realizó una macrocampaña mientras en el otro no hubo campaña alguna, se comprobó que en el estado donde se llevó a cabo la macrocampaña, la población general presentó un incremento de creencias y actitudes adecuadas ante el dolor e igualmente dentro de los especialistas en medicina se mejoró su forma de tratar la lumbalgia (Jolley, Buchbinder y Wyatt, 2001). Debemos considerar estos resultados como una invitación a profundizar en la prevención.

Como ya hemos comentado, en la mayoría de las ocasiones los tratamientos se aplican cuando el paciente padece ya dolor crónico, pero también empiezan a aparecer, como acabamos de señalar, estudios que indican la efectividad de los tratamientos preventivos (Gómez y Méndez, 2000; Miro, 2001). Aparte de estos dos momentos, creemos que existen otros en los que también sería útil aplicar tratamientos interdisciplinares. Por ejemplo, otro momento en el que podrían resultar eficaces sería antes de las intervenciones quirúrgicas que en muchos casos se suelen practicar (por ejemplo en las lumbalgias crónicas). Gatchel (2001) propone una técnica de tratamiento y evaluación de aspectos psicológicos que es eficaz para maximizar los resultados de la cirugía e incluso puede ayudar a tomar decisiones sobre la pertinencia de las intervenciones quirúrgicas en cada caso. Igualmente, Phelan y colaboradores, diseñaron un DVD y un folleto informativo para presentar las distintas opciones terapéuticas (cirugía, medicación...) para aquellas personas que padecían lumbalgia con el objetivo de que tuvieran más información para decidirse o no a ser intervenidos quirúrgicamente (Phelan et al., 2001).

El futuro del tratamiento del dolor, no cabe duda, se basará en la aplicación de programas interdisciplinares. Mientras avanzamos en este camino, todavía quedan multitud de preguntas por responder y cuestiones en las que ahondar como se ha podido empezar a vislumbrar en este apartado. 


\section{Conclusiones}

En el presente artículo hemos intentado analizar los factores psicológicos más importantes que afectan tanto al dolor como a la discapacidad, articulándolos en un esquema teórico basado principalmente en el modelo de estrés de Lazarus. Aunque la causa del dolor sea, en la mayoría de los casos, inicialmente de tipo orgánico parece que los factores psicológicos juegan un papel clave tanto para predecir la discapacidad que el dolor provocará, como su posible cronificación. Creemos que el esquema teórico de Lazarus, tan útil en el campo del estrés, resulta igualmente útil en el campo del dolor. Y aunque en muchas investigaciones en esta línea queda más o menos implícita la utilización de este modelo, queremos remarcar la necesidad de que explícitamente se enmarquen las investigaciones en un mismo modelo, para de esta forma poder corroborarlo, modificarlo, ampliarlo, en definitiva mejorarlo. Una de las razones, por las que consideramos este modelo adecuado en la investigación del proceso doloroso, se basa en que todos los factores psicológicos que se han analizado dentro de la literatura se pueden enmarcar dentro del mismo. Concretamente, las variables estudiadas en este campo pueden ser comprendidas dentro de dos variables clave en este modelo como son la evaluación cognitiva y las estrategias de afrontamiento. Y los demás factores estudiados en esta área (historia previa, psicopatologías, etc.) pueden explicar su influencia sobre el dolor a través de su incidencia sobre estas dos variables.

La aplicación práctica de este esquema teórico debería consistir en su utilización como guía durante el diseño de terapias. Esto es, este modelo debería tenerse en cuenta para que durante las terapias no se dejaran de modificar factores psicológicos que parecen importantes. No siempre las terapias parten de modelos teóricos o de conclusiones de investigaciones más básicas. Por ejemplo, como ya hemos comentado anteriormente, Keefe y colaboradores, señalan la existencia de muchas investigaciones que muestran la relación entre la no expresividad emocional y el dolor (Keefe, Lumbley, Anderson, Lynch y Carson, 2001). Sin embargo, según estos autores son pocos los estudios que utilizan técnicas basadas en la conciencia y expresión de emociones. Por ello, creemos que es conveniente disponer de revisiones teóricas como la que hemos llevado a cabo para que no omitamos durante el tratamiento variables psicológicas clave en el proceso del dolor.

La relación entre los modelos teóricos y las técnicas terapéuticas debe ser bidireccional. No sólo las técnicas deben partir de los modelos teóricos, sino que durante la aplicación de las mismas se debe investigar tanto su efectividad como las variables que modulan dicha efectividad para de esta forma contrastar su sustento teórico. Un claro ejemplo de este tipo de investigación sería el estudio realizado por Jensen y colaboradores, en el que se evaluaron tanto las creencias, como el catastrofismo, las estrategias de afrontamiento y varios parámetros de discapacidad en un grupo de 141 pacientes de un programa multidisciplinar (Jensen, Turner y Romano, 2001). Los resultados mostraron que las mejorías mostradas tras la terapia correlacionaban con los cambios en las creencias y catastrofismo. Concretamente, los pacientes que mostraron más 
mejoría fueron los que disminuyeron sus pensamientos catastrofistas, aumentaron las creencias de control sobre el dolor, y disminuyeron la creencia de que el dolor señaliza daño tisular. Existen otras investigaciones en esta misma línea que sustentan estos resultados (Burns, Kubilus, Bruehl, Harden y Lofland, 2003). Así, este tipo de estudio permite dar sustento a los modelos cognitivosconductuales como el presentado en este artículo.

En este trabajo hemos enfatizado la importancia de las variables psicológicas como factores explicativos del dolor y la discapacidad. Podríamos pensar que el análisis psicológico del dolor que hemos llevado a cabo en estas páginas es reduccionista al no tener en cuenta los factores biológicos. No incluir estos factores en el presente análisis no implica que consideremos el papel de las variables psicológicas más determinante que el de los factores biológicos. Simplemente, el estudio de los factores biológicos no ha sido objeto de nuestro trabajo

Para finalizar solamente queremos resaltar que, aunque la abundante literatura sobre la relación entre factores psicológicos y dolor es más que concluyente, desgraciadamente la aplicación de tratamientos auténticamente interdisciplinares es escasa (Loeser y Melzack, 2001), especialmente en nuestro país. Por este motivo, desde aquí queremos animar a que se diseñen y apliquen este tipo de terapias en nuestras clínicas y hospitales.

\section{REFERENCIAS}

Ashburn, M. A. y Staats, P. S. (1999). Pain: management of chronic pain. Lancet, 353, 186569 [pubmed]

Bishop, K. L., Ferraro, F. R. y Borowiak, D. (2001). Pain management in older adults: role of fear and avoidance. Clinical Gerontologist, 23 (1-2), 33-42.

Biurrun, A. y Jusué, G. (1998). Presentación de un protocolo de tratamiento psicológico (grupal cognitivo-conductual) en pacientes con dolor crónico en Navarra. Anales del Sistema Sanitario de Navarra, 21 (1), 1-10.

Brosschot, J. F. (2002). Cognitive-emotional sensitization and somatic health complaints. Scandinavian Journal of Psychology, 43 (2), 113-121.

Buer, N. y Linton, S. J. (2002). Fear-avoidance beliefs and catastrophizing: ocurrence and risk factor in back pain and ADL in the general population. Pain, 99 (3), 485-491.

Burns, J. W., Kubilus, A., Bruehl, S., Harden, R. N. y Lofland, K. (2003). Do changes in cognitive factors influence outcome following multidisciplinary treatment for chronic pain? A cross-lagged panel analysis. Journal of Consulting and Clinical Psychology, 71 (1), 81-91.

Camacho, L. y Anarte, M. T. (2001). Importancia de las creencias en la modulación del dolor crónico: concepto y evaluación. Apuntes de Psicología, 19 (3), 453-470.

Carmona, L., Ballina, J., Gabriel, R. y Laffon, A. (2001). The burden of musculoskeletal diseases in the general population of Spain: results from a national survey. Annals of the Rheumatic Diseases, 60 (11), 1040-1045.

Casado, M. I. y Urbano, M. P (2001). Emociones negativas y dolor crónico. Ansiedad y Estrés, 7 (2-3), $273-282$.

Català, E. (2002). Prevalence of pain in the Spanish population: Telephone survey in 5000 homes. European Journal of Pain, 6 (2), 133-140.

Cipher, D. J., Fernández, E. y Clifford, P. A (2001). Cost-effectiveness and health care utilization in a multidisciplinary pain center: comparison of three treatment groups. Journal of Clinical Psychology in Medical Settings, 8 (4), 237-244.

Claiborne, N. (2002). Measuring quality of life changes in individuals with chronic low back conditions: a back education program evaluation. Evaluation and Program Planning, 25 (1), 61-70.

Collado, A., Torres, X., Arias, A., Cerad, D., Vilarrasa, R., Valdés, M. y Muñoz-Gómez, J. (2001). Eficacia del tratamiento multidisciplinario del dolor crónico incapacitante del aparato locomotor. Medicina Clínica, $117,401-405$. 
Comeche, M. I. y Lasa, A. (2001). Aspectos cognitivos de la percepción del dolor. Actualizaciones en Dolor, 2 (4), 262-269.

Craufurd, D. I. O., Creed, F., Jayson, M. I. V. (1990). Life events and psychological disturbance in patients with low-back pain. Spine, 15, 490-494.

Crombez, G. (2002). The effects of catastrophic thinking about pain on attentional interference by pain: no mediation of negative affectivity in healthy volunteers and in patients with low back pain. Pain Research and Management, 7 (1), 31-44.

Currie, S. R., Wilson, K. G. y Curran, D. (2002). Clinical significance and predictors of treatment response to cognitivebehaviour therapy for insomnia secondary to chronic pain. Jornal of Behavioral Medicine, 25 (2), 135-153.

De Benedittis G., Lorenzetti, A. y Pieri, A. (1990). The role of stressfull life events in the onset of chronic primary headache. Pain, 40, 65-75.

Dersh, J., Polatin, P. B. y Gatchel, R. J. (2002). Chronic pain and psychopathology: research findings and theoretical consideration. Psychosomatic Medicine, 64(5), 773-786.

Díaz-Sibaja, M. A., Martín, A. y Díaz-García, H.I. (2001). Aspectos comportamentales del dolor crónico. Actualizaciones en Dolor, 2(4), 270-278.

Esteban, S. (1999). El dolor crónico y su abordaje interdisciplinar desde una residencia asistida. Dolor, 14, 193-210.

Esteve, R., López, A. E. y Ramírez, C. (1999). Evaluación de estrategias de afrontamiento al dolor crónico. Journal of Health Psychology, 11 (1-2), 77-102.

Esteve, R., Ramírez, C. y López, A. E. (2001). Aspectos emocionales en el dolor. Actualizaciones en Dolor, 2(4), 252-261.

Esteve, R. y Ramírez, C. (2003). El desafio del dolor crónico. Málaga: Aljibe.

Finestone, H. M., Stenn, P., Davies, F., Stalker, C., Fry, R. y Koumanis, J. (2000). Chronic pain and health care utilization in women with a history of childhood sexual abuse. Child Abuse and Neglect, 24 (4), 547-556.

Fishbain, D. A. (2000). Risc for violent behavior in patients with chronic pain: evaluation and management in the pain facility setting. Pain Medicine, 1 (2), 140-155.

Fishbain, D. A. (2002). The pain-depression relationship. Psychosomatics, 43 (3), 341.

Fisher, B. J., Haythornthwaite, J. A., Heinberg, L. J., Clark, M. y Reed, J. (2002). Intención de suicidio en pacientes con dolor crónico. Revista de la Sociedad Española del Dolor, 9, 146-156.

Flor, H., Knost, B. y Birbaumer, N. (2002). The role of operant conditioning in chronic pain: an experimental investigation. Pain, 95 (1-2), 111-118.

Fordyce, W., Fowler, R. y DeLateur, B. (1968). An application of behavior modification technique to a problem of chronic pain. Behavior Research and Therapy, 6, 105-107.

Gatchel, R.J. (2001). A biopsychosocial overview of pretreatment screening of patients with pain. Clinical Journal of Pain, 17 (3), 192-199.

Gay, M. C. (2002). Differential effectiveness of psychological interventions for reducing osteoarthritis pain: A comparison of Erickson hypnosis and Jacobson relaxation. European Journal of Pain, 6 (1), 1-16.

Goldber, R. T y Goldstein, R. (2000). A comparison of chronic pain patients and controls on traumatic events in childhood. Disability and Rehabilitation: An International Multidisciplinary Journal, 22 (17), 756-763.

González, F. (1999) ¿Es el dolor crónico un problema psiquiátrico aún no clasificado? La fibromialgia. Revista Electrónica de Psiquiatría; 3 (3), 1-21.

Gómez, A. y Méndez, F. X. (2000). Aspectos actuales en la prevención de las lumbalgias. Psicologia Conductual; 8 (1), 99-115.

Górriz, F., Garrido, E. y Andueza, A. (1999). El tratamiento psicológico del dolor crónico. Huarte de San Juan. Psicología y Pedagogía, 4-5, 175-196.

Grant, L. D., Long, B. C. y Willms, J. D. (2002). Women's adaptation to chronic back pain: daily appraisals and coping strategies, personal characteristics and perceived spousal responses. Journal of Health Psychology, 7 (5), 545-564.

Green, C. R., Flowe-Valencia, H., Rosenblum, L. y Tait, A. R. (2001). The role of childhood and adulthood abuse among women presenting for chronic pain management. Clinical Journal of Pain, 17 (4), 359-364.

Grisart, J. M. y Vanderlinden, M. (2001). Conscious and automatic uses of memory in chronic pain patients. Pain, 94 (3), 305-313.

Hawkins, R. (2001). A systematic meta-review of hypnosis as an empirically supported treatment for pain. Pain Reviews, 8, 47-73.

Jansen, S. A. (2002). Negative affect and sensitization to pain. Scandinavian Journal of Psychology, 43 (2), 131-138.

Jensen, M. P., Turner, J. A., Romano, J. M. y Karoly, P. (1991). Coping with chronic pain: a critical review of the literature. Pain, 47 (3), 249-283.

Jensen, M. P., Turner, J. A. y Romano, J. M. (2001). Changes in beliefs, catastrophing, and coping are associated with improvement in multidisciplinary pain treatment. Journal of Consulting and Clinical Psychology, 69 (4), 655-662. 
Jolley, D., Buchbinder, R. y Wyatt, M. (2001). Population based intervention to change back pain beliefs and disability: three part evaluation. British Medical Journal, 7301 (322), 1516-1520.

Keefe, F. J., Lumley, M., Anderson, T., Lynch, T. y Carson, K. (2001). Pain and emotion: new research directions. Journal of Clinical Psycholy, 57 (4), 587-607.

Kerns, R. D., Rosenberg, R. y Otis, J. D. (2002). Self-appraised problem solving and pain-relevant social support as predictors of the experience of chronic pain. Annals of Behavioral Medicine, 24 (2), 100-105.

Lamberg, L (2002). Effective pain treatment promotes activities. Journal of the American Medical Associtation, 288 (8), 948-949.

Lampe, A., Sollner, W., Krismer, M., Rumpold, G., Kantner Rumplmair, W., Ogon, M. y Rathner, G. (1998). The impact of stressful life events on exacerbation of chronic low-back pain. Journal of Psychosomatic Research, 44 (5), 555-563.

Lazarus, R. S. y Folkman, S. (1986). Estrés y procesos cognitivos. Barcelona: Martínez Roca.

Lebovits, A. (2002). Psychological issues in the assessment and management of chronic pain. Annals of the American Psychotherapy Assotiation, 5 (3), 19-21.

Linton, S. y Ryberg, M. (2001). A cognitive-behavioral group intervention as prevention for persistent neck and back pain in a non-patient population: a randomized controlled trial. Pain, 90 (1-2), 83-90.

Loeser, J. D. y Melzack, R. (1999) Pain an overview. Lancet, 353 (91648), 1607-1609.

Martín-Aragón, M., Pastor, M. A., Rodríguez-Marín, J., March, M. J., Lledó, A., López-Roig, S. y Terol, M. C. (1999). Percepción de autoeficacia en dolor crónico. Adaptación y validación de la Chronic pain selfefficacy scale. Revista de Psicología de la Salud, 11 (1-2), 53-75.

Mas, B. y Comeche, M. I. (2001). Aspectos psicofisiológicos implicados en el dolor crónico. Actualizaciones en Dolor, 2(4), 241-251.

Mayr, C. R., Rose, M. J. y Johnstone, F. C. W. (2000). Dealing with doubt: how patients account for nonspecific chronic low back pain. Journal of Psychosomatic Research, 49(4), 223-225.

Mayr, M., Hogler, S., Ghedina, W. y Berek, K. (2003). Low back pain and psychiatric disorders. Lancet, 361, 9356 [pubmed].

McBeth, J., Morris, S., Benjamin, S., Silman, A. J. y MacFarlane, G. J. (2001). Associations between adverse events in childhood and chronic widespread pain in adulthood: are they explained by differential recall? Journal of Rheumatology, 28 (10), 2305-2309.

McCracken, L. M., Goetsch, V. L. y Semenchuk, E. M. (1998). Coping wiht pain produced by physical activity in persons with chronic low back pain: immediate assessment following a specific pain event. Behavioral Medicine, 24 (1), 29-34.

Melzack, R. y Wall, P. D. (1965). Pain mechanism: a new theory. Science, 150, 971-979.

Middaugh, S. J. y Pawlick, K. (2002). Biofeedback and behavioral treatment of persistent pain in the older adult: a review and a study. Applied Psychophysiology and Biofeedback, 27 (3), 185-202.

Miró, J. (2001). Dolor crónico: el reto de la prevención. Revista de Psicología de la Salud, 13 (1), 25-46.

Moix, J. (1990). Estrategias de afrontamiento: consideraciones críticas sobre su concepto y formas de medición. Psicologemas, 4 (8), 195-205.

Moix, J. (2002). La hipnosis en el tratamiento del dolor. Revista de la Sociedad Española del Dolor, 9, $525-532$.

Moix, J. (2003). Eficacia de un programa educativo interdisciplinar en pacientes con dolor de espalda crónico. Dolor: Investigación, Clínica y Terapéutica, 18 (3), 149-157.

Moore, J. E., Von-Korff, M., Cherkin, D., Saunders, K. y Lorig, K. (2000). A randomized trial of a cognitivebehavioral program for enhancing back pain self care in a primary care. Pain, 88 (2), 145-153.

Pastor, M. A., Martín-Aragón, M. y Lledó, A. (1999). Expectativas de autoeficacia y estado de salud en enfermos con dolor crónico: una revisión. Revista de Psicología Social Aplicada, 9 (2), 111-125.

Pastor, M. A., Pons, N., Lledó, A., Martín-Aragón, M., López-Roig, S., Terol, M. C. y Rodríguez-Marín, J. (2003). Evaluación de la eficacia de las intervenciones psicológicas en enfermedades reumáticas: el caso de la fibromialgia. En M. Pérez Álvarez, J.R. Fernández Hermida, C. Fernández Rodríguez e I. Amigo Vázquez (Coords.), Guía de tratamientos psicológicos eficaces. Madrid: Pirámide

Phelan, E. A., Deyo, R. A., Cherkin, D. C., Weinstein, J. A., Ciol, M. A., Kreuter, W. y Howe, J. F. (2001). Helping patients decide about back surgery: a randomized trial of an interactive video program. Spine, 26 (2), 206-211.

Pincus, T. y Morley, S. (2001). Cognitive-processing bias in chronic pain: a review and integration. Psychological Bulletin, 127 (5), 599-617.

Ramírez, C. (2002). Las características personales de la experiencia de dolor y en el proceso de afrontamiento. Escritos de Psicología, 6, 40-52.

Ramírez, C., Esteve, R. y López, A. E. (2001). Neuroticismo, afrontamiento y dolor crónico. Anales de Psicologia, 17 (1), 129-137.

Reeves, J. L. (2000). Depression determines illness conviction and pain impact: a structural equation modeling analysis. Pain Medicine, 1 (3), 238-246.

Anuario de Psicología, vol. 36, $\mathrm{n}^{\circ}$ 1, abril 2005, pp. 37-60

(C) 2005, Universitat de Barcelona, Facultat de Psicologia 
Riley, J., Robinson, M. E., Wade, J. B., Myers, C. D. y Price, D. D. (2001). Sex differences in negative emotional responses to chronic pain. Journal of Pain, 2, 354-359.

Rodríguez-Parra, M. J., Esteve, R. E. y López, A. E. (2000). Dolor crónico y estrategias de afrontamiento. Análisis y Modificación de Conducta, 26 (107), 391-418.

Roelofs, J., Peters, M. L., Zeegers, M. P. y Vlaeyen, J. W. (2002). The modified Stroop paradigm as a measure of selective attention towards pain-related stimuli among chronic pain patients: a meta-analysis. European Journal of Pain, 6 (4), 273-281.

Romano, J. M., Jensen, M. P., Turner, J. A., Good A. B. y Hops, H. (2000). Chronic pain patient-partner interactions: further support for a behavioral model of chronic pain. Behavior Therapy, 31, 415-440.

Roth, R. S. y Geisser, M. E. (2002). Educational achievement and chronic pain disability: mediating role of painrelated cognitions. Clinical Journal of Pain, 18 (5), 286-296.

Serrano, M., Cañas, A., Serrano, C., García, P. L. y Caballero, J. (2002). Evaluación psíquica del dolor. Dolor, $17,57-70$.

Severeijns, R., Vlaeyen, J. W., Van Den Hout, M. A. y Weber, W. E (2001). Pain catastrophizing predicts pain intensity, disability, and psychological distress independent of the level of physical impairment. Clinical Journal of Pain, 17 (2), 165-172.

Sharp, T. J. (2001). Chronic pain: a reformulation of the cognitive-behavioural model. Behaviour Resarch and Therapy, 39, 787-800.

Strong, J., Westbury, K., Smith, G., Mckenzie, I. y Ryan, W. (2002). Treatment outcome in individuals with chronic pain: is the pain stages of change questionnaire a useful tool? Pain, 97 (1-2), 65-73.

Sufka, K. J. (2000). Chronic pain explained. Brain and Mind, 1 (2), 155-179.

Sullivan, M. J. L, Bishop, S.R. y Pivik, J. (1995). The pain catastrophing scales: development and validation. Psychological Assessment, 7 (4), 524-532.

Sullivan, M. J. L., Sullivan, M. E. y Adams, H. M. (2002). Stage of chronicity and cognitive correlates of painrelated disability. Cognitive Behavior Therapy, 32 (3), 111-118.

Tan, G., Jensen, M. P., Robinson, W. S., Thornby, J. I. y Monga, T. N. (2001). Coping with chronic pain: a comparison of two measures. Pain, 90 (1-2), 127-133.

Thorn, B. E., Bothby, J. L. y Sullivan, M. J. L. (2002). Targeted treatment of catastrophizinz for the management of chronic pain. Cognitive and Behavioral Practice, 9 (2), 127-138.

Thunberg, K. A., Carlsson, S. G. y Hallberg, L. R. M. (2001). Health care professionals' understanding of chronic pain: a grounded theory study. Scandinavian Journal of Caring Sciences, 15 (1), 99-105.

Turk, D. C. (2001a). Combining somatic and pscyhosocial treatment for chronic pain patients: perhaps $1+1$ does=3. Clinical Journal of Pain, 17 (4), 281-283.

Turk, D. C. (2001b). Treatment of chronic pain: clinical outcomes, cost-effectiveness and cost benefits. DrugBenefic Tredns, 13 (9), 36-38.

Turk, D. C. y Okifuji, A. (2002). Psychological factors in chronic pain: evoluation and revolution. Journal of Consulting and Clinical Psychology, 70 (3), 678-690.

Turner, J. A., Jensen, M. P. y Romano, J. M. (2000). Do beliefs, coping, and catastrophizing independently predict functioning in patients with chronic pain? Pain, 85, 115-125.

Turner, J. A., Jensen, M. P., Warms, C. A. y Cardenas, D. D. (2002). Catastrophizing is associated with pain intensity, psychological distress, and pain-related disability among indiviudals with chronic pain after spinal cord injury. Pain, 98 (1-2), 127-134.

Vallejo, M. A. y Comeche M. I. (1994). Evaluación y tratamiento psicológico del dolor crónico. Madrid: Fundación Universidad-Empresa.

Van-den-hout, J. H. C.,Vlaeyen, J. W. S., Heuts, P. H. T. G., Sillen, W. J. T. y Willen, A. J. E. (2001). Functional disability in nonspecific low back pain: the role of pain-related fear and problem-solving skills. International Journal of Behavioral Medicine, 8 (2), 134-148.

Vlaeyen, J. W., De Jong, J. R., Onghena, P., Kerkhoffs-Hanssen, M. y Kole-Snijders, A. M. (2002). Can painrelated fear be reduced? The application of cognitive-behavioral exposure in vivo. Pain Research and Management, 7 (3), 144-153.

Von-Korff, M., Moore, J. E., Lorig, K., Cherkin, D. C., Saunders, K., Gonzalez, V. M., Laurent, D., Rutter, C. y Comite, F. (1998). A randomized trial of a lay person-led self-management group intervention for back pain patients in primary care. Spine, 23, 2608-2615.

Walsh, D. A. y Radcliffe, J. C. (2002). Pain beliefs and perceived physical disability of patients with chronic low back pain. Pain, 97 (1-2), 23-31.

Witty, T. E., Heppner, P. P., Bernard, C. B. y Thoreson, R. W. (2001). Problem-solving appraisal and psychological adjustment of persons with chronic low-back pain. Journal of Clinical Psychology in Medical Setting, 8 (3), 149-160. 